\title{
The Efficacy of Optimum Apgar Scoring System For Prediction of Neonatal Mortality and Morbidities in Preterm Infants
}

\section{Gokhan Buyukkale, Emre Dincer, Merih Cetinkaya
*Health Sciences University, Kanuni Sultan Suleyman Training and Research Hospital, Department of Neonatology, Istanbul, Turkey

Introduction: As conventional Apgar system was suggested to be limited use in preterm infants, Combined Apgar system was defined with the inclusion of cord blood gas $\mathrm{pH}$ and targeted oxygen saturation levels as a beter tool. Our group developed Optimum Apgar scoring system by the exlusion of two criteria (appearance and grimace) as a more objective tool for evaluation of premature infants in the delivery room. The aim of this study was to evaluate the utility of Optimum Apgar Scoring System for prediction of neonatal morbidities and mortality in the first week of life in very low birth weight (VLBW) infants and to compare its' efficacy with both Conventional and Combined Apgar scoring systems.

Methods: This prospective cohort study was performed in the delivery room between January 2017 and December 2017. The inborn premature infants with a gestational age $<32$ weeks and admitted to neonatal intensive care unit were included. All infants were evaluated by Conventional, Combined and Optimum scores at $1^{\text {st }}$ and $5^{\text {th }}$ minutes. For Optimum scoring system, blood sample was obtained from the umbilical artery and pulse oximetry was used for measurement of oxygen saturation. The demographical data, neonatal morbidities and mortality in the first week of life were all recorded.

Results: A total of 589 VLBW infants were included. The mean gestational age and birth weight of these infants were $27.3 \pm 2 \mathrm{w}$ and $890 \pm 212 \mathrm{~g}$. The median Conventional, Combined and Optimum Apgar scores of infants at $1^{\text {st }}$ and $5^{\text {th }}$ minutes were (5-7), $(9-11)$ and $(8-10)$, respectively. From these, 52 infants died in the first week of life. The infants who died had significantly lower gestational age and birth weight compared with those survived. Although the $1^{\text {st }}$ minute scores of all the 3 scoring systems were lower in infants who died, the median Combined and Optimum Apgar Scores at minute 5 were significantly lower (for both; $p=0.001$ ). For prediction of mortality, the sensitivity and specifivity of $5^{\text {th }}$ minute Combined and Optimum Apgar scores were found as (73\% and $82 \%$ ) and ( $70 \%$ and $84 \%$ ), respectively. The cut off values of $5^{\text {th }}$ minute Combined and Optimum Apgar scores for prediction of mortality were $\leq 9$ and $\leq 7$, respectively. The Combined and Optimum Apgar scores at minute 5 were significantly lower in infants that developed PDA, ROP, NEK and IVH (for both; $p<$ 0.05).

Conclusion: To our best of knowledge, the efficacy of Optimum Apgar scoring system was evaluated for the first time. We suggest that Optimum Apgar scoring system may be used as a more accurate and objective tool for prediction of neonatal morbidities and mortality in in the first week of life in VLBW infants. It may be considered as a more reliable scoring system for evaluation of VLBW infants in the delivery room. However, further studies with larger number of infants are required to elucidate its' utility in preterm infants. 